\title{
Assessment of hematopoietic failure due to Rpl11 deficiency in a zebrafish model of Diamond- Blackfan anemia by deep sequencing
}

Zhaojun Zhang ${ }^{1 \dagger}$, Haibo $\mathrm{Jia}^{2+}$, Qian Zhang ${ }^{1}$, Yang Wan ${ }^{3}$, Yang Zhou ${ }^{2}$, Qiong Jia ${ }^{2}$, Wanguang Zhang ${ }^{4}$, Weiping Yuan ${ }^{3}$, Tao Cheng ${ }^{3}$, Xiaofan Zhu ${ }^{3^{*}}$ and Xiangdong Fang ${ }^{1^{*}}$

\begin{abstract}
Background: Diamond-Blackfan anemia is a rare congenital red blood cell dysplasia that develops soon after birth. RPL11 mutations account for approximately $4.8 \%$ of human DBA cases with defective hematopoietic phenotypes. However, the mechanisms by which RPL11 regulates hematopoiesis in DBA remain elusive. In this study, we analyzed the transcriptome using deep sequencing data from an Rpl11-deficient zebrafish model to identify Rpl11-mediated hematopoietic failure and investigate the underlying mechanisms.

Results: We characterized hematological defects in Rpl11-deficient zebrafish embryos by identifying affected hematological genes, hematopoiesis-associated pathways, and regulatory networks. We found that hemoglobin biosynthetic and hematological defects in Rpl11-deficient zebrafish were related to dysregulation of iron metabolism-related genes, including tfa, tfr1b, alas2 and s/c25a37, which are involved in heme and hemoglobin biosynthesis. In addition, we found reduced expression of the hematopoietic stem cells (HSC) marker cmyb and HSC transcription factors tal1 and hoxb4a in Rpl11-deficient zebrafish embryos, indicating that the hematopoietic defects may be related to impaired HSC formation, differentiation, and proliferation. However, Rpl11 deficiency did not affect the development of other blood cell lineages such as granulocytes and myelocytes.
\end{abstract}

Conclusion: We identified hematopoietic failure of Rpl11-deficient zebrafish embryos using transcriptome deep sequencing and elucidated potential underlying mechanisms. The present analyses demonstrate that Rpl11-deficient zebrafish may serve as a model of DBA and may provide insights into the pathogenesis of mutant RPL11-mediated human DBA disease.

Keywords: Zebrafish, Hematopoiesis, Rpl11, RNA-Seq, Transcriptome, DBA

\section{Background}

Ribosomal protein dysfunction causes abnormal ribosomal particle assembly and affects protein synthesis in all cell types. Although RP dysfunction affects multiple tissues and systems, pathological defects in hematopoietic systems, such as aberrant development in Diamond-Blackfan

\footnotetext{
*Correspondence: zhuxf6465@gmail.com; fangxd@big.ac.cn

${ }^{\dagger}$ Equal contributors

${ }^{3}$ State Key Laboratory of Experimental Hematology, Institute of Hematology and Blood Disease Hospital, Chinese Academy of Medical Sciences \& Peking Union Medical College, Tianjin 300020, China

${ }^{1}$ CAS Key Laboratory of Genome Sciences and Information, Beijing Institute of Genomics, Chinese Academy of Sciences, Beijing 100101, China Full list of author information is available at the end of the article
}

anemia (DBA), are the most frequently observed phenotypes [1-3].

Mutations in RP genes reduce the efficiency of ribosome biogenesis, which is indispensable for immature erythrocytes in early and rapid growth phases [4]. Defective ribosome biosynthesis leads to excessive release of RPs such as RPL5 and RPL11, which may increase p53 activity by activating the RPS MDM2-p53 signaling pathway [5]. Because erythroid progenitor cells are highly sensitive to $\mathrm{p} 53$ activation [6], subsequent activation of the p53 pathway in erythroid progenitor cells leads to cell cycle arrest and apoptosis and finally to hematopoietic disorders. Interestingly, these hematopoietic defects were rescued by increased RP expression or reduced p53 activity in zebrafish

\section{Biomed Central}


and in erythroid progenitor cells from DBA patients [7-10]. It was also observed that Rpl22l1 plays essential roles in hematopoiesis, but acts independently of p53 and does not induce apoptosis [11].

DBA is a rare congenital red blood cell dysplasia that develops soon after birth. This disorder is mainly characterized by reduced reticulocyte counts, selective red cell aplasia, and macrocytosis, while a number of other blood cell lineages, such as neutrophils and platelets, are maintained or slightly decreased. Furthermore, DBA patients suffer other developmental defects, such as short stature, cleft lip, hand deformities, kidney and heart hypoplasia, and tumor predisposition [12-14]. Ribosome biogenesis defects have been identified as a major cause of DBA, and mutations of genes encoding RPs have emerged as the main cause of DBA. Indeed, various mutations have been found in coding and noncoding regions of at least 13 RPs, including RPL5, RPS7, RPL9, RPS10, RPL11, RPS15, RPS17, RPS19, RPS24, RPS26, RPL36, RPS27a, and $R P L 35 a$, accounting for about $55 \%$ of all DBA cases [15-23]. RPS19 was the first identified causative gene in DBA patients, and its mutations account for $25 \%$ of DBA cases [24].

RPL11 is one of 79 vertebrate RPs, and its gene mutations occur on coding and intronic binding regions of chromosome 1 [17]. DBA patients with mutations in RPL11 show typical hematopoietic defects, and $67 \%$ of these have physical deformities, especially of the thumb [17]. In particular, RPL11 dysfunction results in abnormal erythrocyte development, markedly decreased progenitor cell proliferation, delayed erythroid differentiation, and markedly increased apoptosis [25]. Importantly, these are different from the deficiencies in DBA patients with RPS19 mutations [25]. Hence, divergent mechanisms underlie hematopoietic defects in DBA patients with different RP mutations. Moreover, RPL11 dysfunction in erythroblasts lead to aberrant erythroblast differentiation, reduced hemoglobin, and unusual cell morphology [26].

Zebrafish are a classic animal model for mechanistic studies of embryonic development, hematopoiesis, and DBA $[27,28]$. Knockdown of most RP genes causes developmental defects in the brain of zebrafish, indicating that the vast majority of RP functions are associated with nervous system development [29]. Several reports focus on regulation of zebrafish hematopoiesis by the RP proteins Rps7, Rpl11, Rps19, and Rps29 [8-10,30]. Rpl11 dysfunction in zebrafish embryos leads to defective development, hematopoiesis, brain development, and shape dysplasia [8,29,31]. Furthermore, Danilova et al. reported that the hematopoietic defects caused by Rpl11 dysfunction were fully reversed by the inhibition of p53 activity in zebrafish mutants [8], indicating that p53 may regulate Rpl11 in zebrafish [25,31]. However, hematopoiesis in zebrafish with dysfunctional Rps19 is not fully recovered by inhibition of p53 [32]. Presumably, these varying phenotypic defects in zebrafish embryos with dysfunctional Rpl11 are regulated by multifactorial interactions of genes, regulatory networks, and signaling pathways.

High-throughput transcriptome sequencing is now widely accepted as a useful tool for investigating human disease mechanisms and gene functions. However, this technology has not been widely used in zebrafish research. Using microarray technology, Danilova et al. (2011) studied the impact of Rpl11 deficiency on hematopoiesis of zebrafish embryos. However, because of the limitations of detectable gene transcripts, some important aspects of Rpl11 functions in zebrafish may only be elucidated using high-throughput sequencing.

In this study, we examined genome-wide transcription by high-throughput transcriptome sequencing of Rpl11-deficient zebrafish embryos. In combination with bioinformatic analyses, we studied affected genes, hematopoiesis-related signaling pathways, and molecular regulatory networks in Rpl11-deficient zebrafish embryos. We found that Rpl11 deficiency leads to defects of hemoglobin synthesis and hematopoiesis, which were caused by dysregulation of iron metabolism-related genes and impaired HSC differentiation and proliferation. These analyses provide new insights into the pathogenesis of mutant Rpl11-mediated DBA.

\section{Results}

\section{Hematopoietic defects in Rpl11-deficient zebrafish embryos}

To investigate the regulation of Rpl11 in hematopoiesis in zebrafish embryos, we initially established an Rpl11deficient zebrafish model using MO knockdown technology. We observed various phenotypes in this model, including smaller brains, tail deformities, and hematopoietic defects, which were consistent with other studies [8,29,31]. Benzidine staining of Rpl11-deficient zebrafish embryos at 48 hpf showed that globin expression was significantly decreased compared with that in control MO embryos, indicating defects of globin synthesis during late erythroid differentiation (Figure 1A-D). Subsequently, deep sequencing was performed to investigate the impact of Rpl11 on the transcriptome of Rpl11-deficient and control MO embryos at $48 \mathrm{hpf}$. The effectiveness of translational inhibition by Rpl11 MO was confirmed by examining the green fluorescent fusion protein under a fluorescence microscope (Figure 1E-F).

\section{Transcriptome changes in Rpl11-deficient zebrafish embryos}

To comprehensively reflect the impact of Rpl11 deficiency on the transcriptome of zebrafish embryos, we collected 40-50 Rpl11-deficient and MO control zebrafish embryos at $48 \mathrm{hpf}$ from at least three separate experiments and 


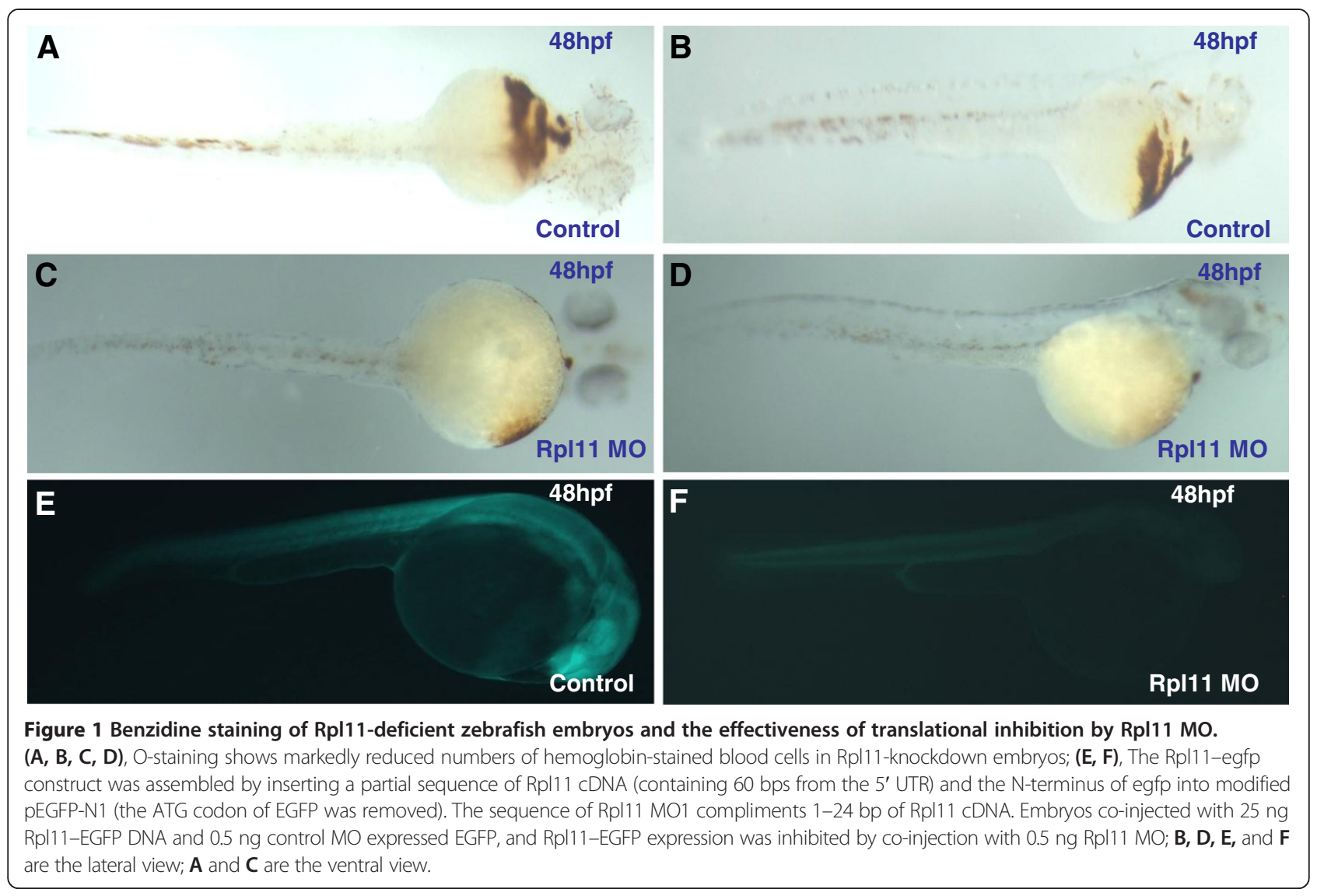

constructed two mRNA-seq sequencing libraries in parallel. High-throughput sequencing was performed on the Hi-Seq2000 sequencing platform in parallel. The number of sequenced gene transcript reads was 35-40 million. Sequencing data were mapped to the latest $\mathrm{Zv} 9$ version of the zebrafish genome. Subsequently, 15-17 million readable gene transcripts were mapped to the zebrafish genome with $41-45 \%$ mapping efficiency (Additional file 1: Table S1). On average, more than 10,000 gene transcripts were mapped to known zebrafish genes, accounting for at least $70 \%$ of annotated genes. The percentage of detected unique gene transcripts was similar to that detected in previous studies [33,34], indicating that the present sequencing represented the vast majority of zebrafish genes.

All sequenced gene transcript reads were standardized to FPKM [35], and gene expression was presented (Additional file 2: Table S2). The $\mathrm{R}$ package DEGseq was used to compare transcriptomes of Rpl11-deficient zebrafish embryos and MO control embryos. In total, we identified 572 significantly differentially expressed genes (DEGs, FC >2, p-value <0.05); 483 were downregulated and 89 were upregulated. We also identified DEGs using screening thresholds set at p-value $<0.05$ and FC >1.5 (Additional file 3: Table S3). In addition, we found 10 specifically expressed genes (p-value $<0.05$ ) that were only expressed in one sample (Additional file 4: Table S4). Among these, four were significantly upregulated in Rpl11-deficient zebrafish embryos, whereas expression of the other six was almost completely inhibited, indicating high dependence of gene expression on Rpl11.

\section{Gene ontology analysis of Rpl11 in zebrafish embryos}

To investigate the biological functions of Rpl11 in zebrafish embryos, we performed GO analyses of genes that were significantly regulated by Rpl11 deficiency using AmiGO software (http://www.geneontology.org/). Since IPA software does not contain the zebrafish gene annotation that must be transformed into human genes before analysis, we used the GO analysis tool in the ZFIN database to get more detailed information of Rpl11 functions in zebrafish. GO analyses showed that upregulated genes were specifically enriched in functions associated with iron metabolism, including iron transport, intracellular iron homeostasis, and iron binding (Additional file 5: Figure S1 and Additional file 6: Table S5). Three zebrafish genes were involved in these iron-related functions, including zgc:194125, zgc:109934, and zgc:173594 (Additional file 7: Figure S3). However, no detailed gene annotations exist in the latest version $(\mathrm{Zv} 9)$ of the zebrafish genome. Interestingly, a single human mitochondrial 
ferritin (FTMT) homolog was found for all three of these zebrafish genes, and its aberrant expression resulted in accumulation of mitochondrial iron, disruption of intracellular iron homeostasis [36], and reduction in iron utilization for heme and hemoglobin synthesis [37]. Downregulated genes were specifically enriched in functions associated with development and differentiation of the nervous system, generation of neurons and brain development, molecular metabolic regulation, developmental regulation of cells and tissues, regulation of gene transcription, regulation of RNA synthesis and metabolism, and other functions (Additional file 5: Figure S2 and Additional file 6: Table S5). Thus, GO analyses demonstrated that Rpl11 participates in the regulation of multiple biological processes in zebrafish embryos, and particularly in hematopoietic iron metabolism-associated pathways.

\section{Hematological genes affected by Rpl11 deficiency in zebrafish embryos}

To further investigate hematopoietic failures caused by Rpl11 deficiency in zebrafish, we analyzed affected hematological genes in Rpl11-deficient zebrafish embryos at $48 \mathrm{hpf}$. To provide a more detailed list of hematological genes affected by Rpl11 deficiency, the screening criteria were set at FC $>1.5$ and p-value $<0.05$. In total, 33 differentially expressed hematological genes were identified (Additional file 8: Figure S4), and description of these genes were summarized in Table 1. Heatmap analyses showed that most (24/33) hematological genes were significantly downregulated by Rpl11 deficiency (Figure 2A). Subsequent GO analyses demonstrated that these hematological genes were specifically enriched in diverse hematopoiesisassociated biological processes, including oxidationreduction processes, heme binding, hypoxic oxygen binding, oxygen transporter activity, iron transport, and oxygen transport (Figure 2B). Hematological genes involved in GO items were summarized in (Additional file 9: Table S6).

\section{Identification of hematopoiesis-associated pathways disturbed in Rpl11-deficient zebrafish}

Key signaling pathways in mammals are highly conserved in zebrafish. To elucidate the roles of Rpl11 in the regulation of hematopoiesis in zebrafish embryos, we used IPA software to identify the signaling pathways that are most affected (p-value <0.01) by Rpl11 deficiency. Separate pathways were constructed with upregulated and downregulated genes (Additional file 10: Table S7). Among the five most affected signaling pathways, we identified three hematopoiesis pathways that were upregulated, including glucocorticoid receptor signaling [38,39], IGF-1 signaling [40-42], and IL-17A signaling in fibroblasts [43], and two hematopoiesis-related pathways that were downregulated, including $\mathrm{Wnt} / \beta$-catenin sig- naling [44-48] and Aryl hydrocarbon receptor signaling [49] (Table 2). Among these pathways, Wnt/ $\beta$-catenin signaling has been characterized as essential for formation, self-renewal, and development of hematopoietic stem cells (HSCs) [48], which are closely associated with hematopoiesis. Taken together, Rpl11 deficiency disturbed hematopoiesis-related signaling pathways in zebrafish embryos, at least partially explaining the ensuing hematopoietic failure.

\section{Identification of regulatory networks affected by Rpl11 deficiency in zebrafish embryos}

We used IPA software to determine whether molecular regulatory networks were disturbed by Rpl11 deficiency in zebrafish embryos (Additional file 11: Figure S5A-C, Figure S6A-E, and Additional file 12: Table S8). Analyses showed no specifically enriched hematopoiesis-associated regulatory networks of differentially regulated genes. Potentially, hematopoiesis-associated networks may have been weakened by more important functions of Rpl11 in zebrafish embryos and were therefore not detectable or were obscured by networks regulating hematopoiesis by unknown mechanisms. Through these networks, Rpl11 may play critical regulatory roles in cell development, proliferation, and apoptosis, nervous system development, embryonic development, immunological disease, and tumor pathology. We also identified central network nodes that may directly or indirectly associate with RPL11, including MMP9, AP1, SERPINE1, CTGF, FOS, JUNB, SOCS3, SOX2, TWIST1, CCND1, MMP2, and APP.

\section{Potential mechanisms through which Rpl11 regulates hematopoiesis in zebrafish embryos}

Benzidine staining of Rpl11-deficient zebrafish embryos showed that hemoglobin biosynthesis was significantly diminished, suggesting defects in hemoglobinization and late erythroid differentiation, which are consistent with the hypochromic anemia typical of iron, heme, or globin deficit in zebrafish embryos [50]. Focusing on the genes associated with erythrocyte iron metabolism, we found that the majority of genes involved in zebrafish iron metabolism were abnormally expressed (Table 3 ). These included alas2, cp, fth1a/b, htt, aco2, slc25a37, sfxn1, tfa, and $t$ fr $1 b$, suggesting that Rpl11 deficiency in zebrafish might affect multiple aspects of iron metabolism that are closely regulated in highly hemoglobinized erythrocytes. For example, reduced expression of $t f a$ and $t f r 1 b$ transcripts indicates damaged iron acquisition by erythrocytes and that of slc25a37 and alas 2 indicates aberrant iron utilization by erythrocytes (Figure 3A). Moreover, upregulation of three homologs of human FTMT in Rpl11-deficient zebrafish embryos, including zgc:194125, $z g c: 109934$, and $z g c: 173594$ (Additional file 7: Figure S3), 
Table 1 Affected hematological genes in Rpl11-deficient zebrafish embryos at 48 hpf

\begin{tabular}{|c|c|c|c|c|}
\hline Genes & Fold change & p-Value & Description & Human homolog \\
\hline hbbe3 & $\uparrow 3.12$ & 0 & Hemoglobin beta embryonic-3 & HBE1 \\
\hline hbbe2 & $\uparrow 1.56$ & $3.84 \mathrm{E}-23$ & Hemoglobin beta embryonic-2 & HBE1 \\
\hline zgc:153284 & $\uparrow 2.32$ & $6.20 \mathrm{E}-13$ & zgc:153284 & - \\
\hline cyp26a1 & $\uparrow 3.13$ & 0.001089 & Cytochrome P450, subfamily XXVIA, polypeptide 1 & CYP26A1 \\
\hline $\mathrm{dd} \times 18$ & $\uparrow 1.58$ & 0.003224 & DEAD (Asp-Glu-Ala-Asp) box polypeptide 18 & DDX18 \\
\hline cyp24a1 & $\uparrow 6.16$ & 0.014658 & Cytochrome P450, family 24, subfamily A, polypeptide 1 & CYP24A1 \\
\hline $\operatorname{ptgs} 2 a$ & $\uparrow 2.21$ & 0.023441 & Prostaglandin-endoperoxide synthase $2 \mathrm{a}$ & - \\
\hline zgc:56493 & $\downarrow 1.63$ & $2.38 \mathrm{E}-18$ & zgc:56493 & TXN \\
\hline $\operatorname{cox} 4 \mathrm{il}_{1}$ & $\downarrow 1.52$ & $6.39 \mathrm{E}-17$ & Cytochrome c oxidase subunit IV isoform 1 & COX411 \\
\hline pdia3 & $\downarrow 2.94$ & $1.45 \mathrm{E}-13$ & Protein disulfide isomerase family A, member 3 & PDIA3 \\
\hline pdia4 & $\downarrow 3.75$ & $7.23 \mathrm{E}-10$ & Protein disulfide isomerase associated 4 & PDIA4 \\
\hline hdac1 & $\downarrow 1.68$ & 8.99E-08 & Histone deacetylase 1 & $\mathrm{HDAC1}$ \\
\hline Cat & $\downarrow 3.66$ & $2.19 \mathrm{E}-06$ & Catalase & CAT \\
\hline rrm2 & $\downarrow 2.04$ & $1.47 \mathrm{E}-05$ & Ribonucleotide reductase M2 polypeptide & - \\
\hline p4hb & $\downarrow 2.02$ & $2.57 \mathrm{E}-05$ & Procollagen-proline, 2-oxoglutarate 4-dioxygenase, beta polypeptide & $\mathrm{P} 4 \mathrm{HB}$ \\
\hline rrm1 & $\downarrow 1.82$ & $3.28 \mathrm{E}-05$ & Ribonucleotide reductase M1 polypeptide & RRM1 \\
\hline сур2аa8 & $\downarrow 2.29$ & 0.000119 & cytochrome P450, family 2 , subfamily AA, polypeptide 8 & - \\
\hline tp53 & $\downarrow 1.66$ & 0.000172 & Tumor protein p53 & TP53 \\
\hline agxt211 & $\downarrow 4.63$ & 0.000349 & Alanine-glyoxylate aminotransferase 2-like 1 & AGXT2L1 \\
\hline pdip5 & $\downarrow 2.06$ & 0.000366 & Protein disulfide isomerase-related protein (provisional) & PDIA6 \\
\hline alas2 & $\downarrow 1.60$ & 0.000435 & Aminolevulinate, delta-, synthetase 2 & ALAS2 \\
\hline Max & $\downarrow 1.88$ & 0.000645 & myc-associated factor $X$ & MAX \\
\hline isca1 & $\downarrow 1.72$ & 0.000755 & Iron-sulfur cluster assembly 1 & ISCA1 \\
\hline sh3bgrl3 & $\downarrow 2.39$ & 0.002398 & SH3 domain binding glutamic acid-rich protein like 3 & SH3BGRL3 \\
\hline pgrme1 & $\downarrow 1.86$ & 0.003269 & Progesterone receptor membrane component 1 & PGRMC1 \\
\hline snrnp70 & $\downarrow 1.76$ & 0.006001 & Small nuclear ribonucleoprotein 70 (U1) & SNRNP70 \\
\hline cyp2aa4 & $\downarrow 2.27$ & 0.007172 & Cytochrome P450, family 2, subfamily AA, polypeptide 4 & - \\
\hline mta2 & $\downarrow 1.62$ & 0.009737 & Metastasis associated 1 family, member 2 & MTA2 \\
\hline sema3d & $\downarrow 4.42$ & 0.016997 & Semaphorin 3d & SEMA3D \\
\hline txndc5 & $\downarrow 1.53$ & 0.029683 & Thioredoxin domain containing 5 & TXNDC5 \\
\hline $\mathrm{Mb}$ & $\downarrow 2.85$ & 0.037113 & Myoglobin & MB \\
\hline slc40a1 & $\downarrow 1.96$ & 0.037939 & Solute carrier family 40 (iron-regulated transporter), member 1 & SLC40A1 \\
\hline pgrmc2 & $\downarrow 1.63$ & 0.038689 & Progesterone receptor membrane component 2 & PGRMC2 \\
\hline
\end{tabular}

Differentially expressed hematological genes in Rpl11-deficient zebrafish embryos were identified (Fold change $>1.5, \mathrm{p}$-value $<0.05$ ).

could lead to imbalances in cytoplasmic and mitochondrial iron metabolism in erythrocytes [37]. At present, it is widely reported that dysfunction of these genes in animal models leads to hematopoiesis-associated diseases such as siderocytic and microcytic anemia (Table 3). Taken together, aberrant expression of these genes may seriously damage iron metabolism, iron supply for heme biosynthesis, and hemoglobin biosynthesis during late erythroid differentiation, finally resulting in defective hematopoiesis in zebrafish embryo.
HSCs are capable of self-renewal and have the potential to differentiate into all blood cell lineages, including erythrocytes. Runt-related transcription factor 1 (Runx1) was previously shown to be necessary for formation of HSCs. However, mRNA expression of runx 1 was too low $($ FPKM $<1)$ to represent its actual expression in zebrafish embryos using RNA-Seq data. Thus, we assessed the proliferation and differentiation of HSCs in Rpl11deficient zebrafish embryos by observing the expression of the HSC marker cmyb. As shown in Figure 3B and 


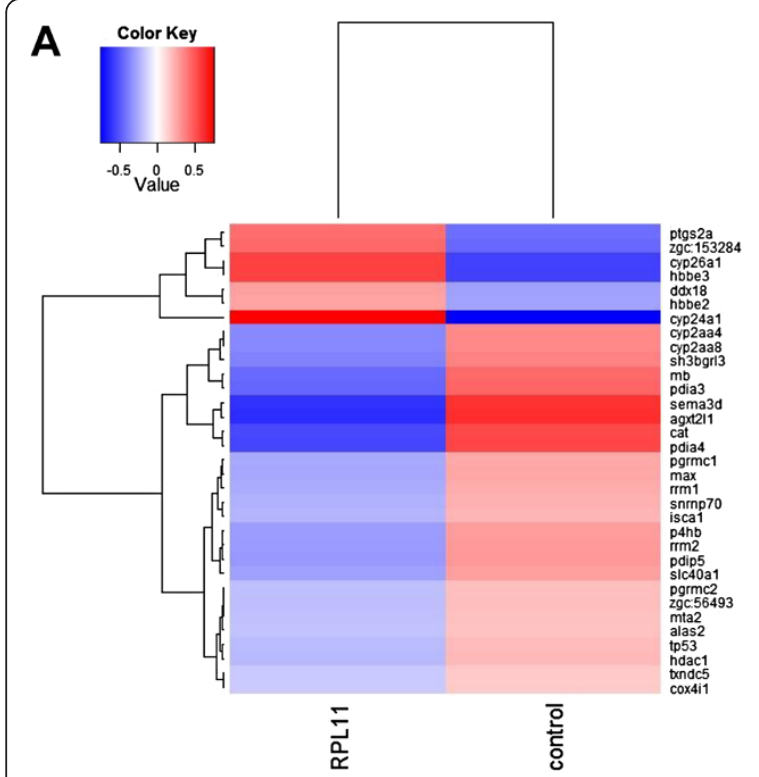

\section{B}

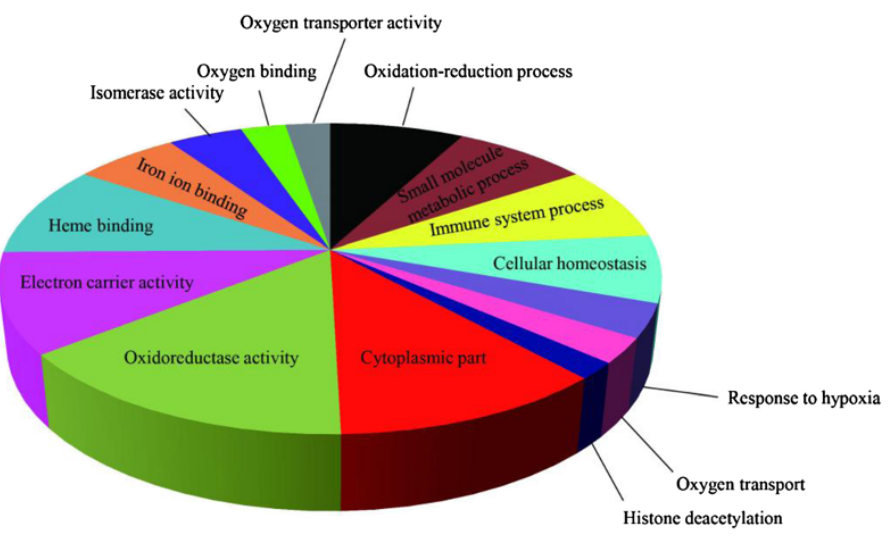

Figure 2 Analysis of affected hematological genes in Rpl11-deficient zebrafish embryos at $\mathbf{4 8}$ hpf. A, Cluster analysis of affected hematological genes. A total of 33 hematological genes exhibited changed expression in Rpl11-deficient zebrafish embryos at 48 hpf (FC >1.5, $\mathrm{p}$-value $<0.05$ ). Hematological genes were clustered in upregulated and downregulated groups, and most affected hematopoietic genes were downregulated in Rpl11-deficient zebrafish embryos at 48 hpf; B, Gene ontology (GO) enrichment of affected hematological genes in Rpl11deficient zebrafish embryos at 48 hpf. Among hematological genes, the largest three fractions represented oxidoreductase activity, cytoplasmic component, and electron carrier activity. Hematological genes with iron metabolism-associated functions such as heme and iron binding were also enriched.

Additional file 13: Table S9, we observed a marked decrease in the mRNA expression of $c m y b$, indicating reduction in HSCs in Rpl11-deficient zebrafish embryos. In addition, we observed dramatic reductions in mRNA expression of HSC transcription factors, including hoxb4a, which regulates HSC expansion [63], and tal1, which controls differentiation and development of HSCs [64]. Given occasional dysplasia of blood cell lineages other than erythrocytes in DBA patients, we determined whether Rpl11 deficiency affected the development and proliferation of granulocytes, lymphocytes, and myelocytes by observing the cellular markers $i k z f 1$, spil, and $m p x$, respectively. qPCR analyses showed no changes in expression of these markers in Rpl11-deficient zebrafish embryos (Figure 3B), suggesting that development and proliferation of lymphocytes, myelocytes, and granulocytes were unaffected by Rpl11 deficiency. Taken together, hematopoietic failure in Rpl11-deficient zebrafish may be attributed to defective erythropoiesis in zebrafish embryo HSCs.

\section{Discussion}

\section{P53 pathway acts independently of Rpl11-deficiency in zebrafish embryos}

In previous studies, hematopoiesis was sensitive to p53 activation [6], and p53 pathway was shown to be activated in DBA patients [25,65] and in RP-deficient zebrafish with defective hematopoiesis $[9,10,30]$. However, we did not observe upregulation of p53 signaling in Rpl11-deficient zebrafish embryos. Potentially slight changes in expression of p53-related genes were obscured by more marked transcriptional consequences of Rpl11 deficiency in zebrafish embryos. However, these observations are consistent with RPL11-independent activation of the p53 pathway in DBA patients [25]. In addition, p53 expression was decreased in tumor cells with dysfunctional RPs [66], suggesting that p53 expression may be sensitive to tumor and tissue environments. Consistent with cells of DBA patients, we speculate that cells of Rpl11-deficient zebrafish embryos may carry characteristics of tumor cells $[13,17,67]$.

It remains unclear whether the entire hematopoietic defective phenotype of DBA is associated with increased expression of p53 or p53-independent mechanisms [25]. Indeed, in a recent report, dysfunctional Rpl22 and Rpl22l1 led to blockage of HSC emergence. Although Rpl2211 plays essential roles in hematopoiesis, it did not induce apoptosis and acted independently of p53 [11]. In this study, we tested the effects of p53 on phenotypes of Rpl11-deficient zebrafish embryos by observing changes in the expression of the HSC formation marker cmyb in Rpl11 morphants and Rpl11 and p53 in double morphants at $48 \mathrm{hpf}$. In these experiments, decreased cmyb expression was not significantly rescued by p53 $\mathrm{MO}$ at 
Table 2 Affected hematopoiesis-associated signaling pathways in Rpl11-deficient zebrafish embryos at 48 hpf

\begin{tabular}{|c|c|c|c|c|}
\hline Ingenuity canonical pathways & -log (p-value) & Up/down regulation & Functions & Reference \\
\hline Wnt/ß-catenin signaling & $3.81 \mathrm{E}+00$ & Down & HSC formation, self-renewal, and differentiation & {$[44-48]$} \\
\hline Aryl hydrocarbon receptor signaling & $3.70 E+00$ & Down & Maintenance of HSC quiescence & [49] \\
\hline IGF-1 signaling & $4.28 \mathrm{E}+00$ & Up & $\begin{array}{l}\text { Regulation of hematopoiesis and proliferation of } \\
\text { hematopoietic stem cell progenitor cells }\end{array}$ & {$[40-42]$} \\
\hline IL-17A signaling in fibroblasts & $4.27 \mathrm{E}+00$ & Up & $\begin{array}{l}\text { Linking T cell function and hematopoiesis, mediating } \\
\text { the hematopoietic response }\end{array}$ & [43] \\
\hline Glucocorticoid receptor signaling & $3.67 \mathrm{E}+00$ & Up & $\begin{array}{l}\text { Stimulation of BFU-E progenitor self-renewal } \\
\text { and regulation of stress erythropoiesis }\end{array}$ & {$[38,39]$} \\
\hline
\end{tabular}

the base of the Rpl11 MO construct (Figure 4A-C). In addition, we also tested the effects of p53 on defective hematopoietic phenotypes of Rpl11-deficient zebrafish embryos by performing hemoglobin staining of embryos injected with Rpl11 MO and Rps19 MO, both individually and with coinjections of p53 MO. O-staining showed a significant reduction in the number of hemoglobin stained blood cells in individual Rpl11 or Rps19 knockdowns. The Rps19 MO phenotype was partially rescued by coinjection with p53 MO, whereas the Rpl11 MO phenotype was not (Figure 4D-I). Taken together, these results demonstrated that Rpl11 leads to hematopoietic defects in zebrafish embryos through p53-independent pathways.

\section{Hemoglobin genes' translation could be inhibited in Rpl11-deficient zebrafish embryos}

We observed markedly reduced hemoglobin expression in Rpl11-deficient zebrafish embryos and analyzed globin expression using RT-PCR analysis data (Additional file 14: Figure S7). In total, we identified six globin transcripts hbaa1, hbae1, hbae3, hbbe1, hbbe2, and hbbe3 in zebrafish embryos at $48 \mathrm{hpf}$ in RNA-Seq data. Among these, hbae1, hbae3, hbbe1, and hbbe3 are specifically expressed in embryos, while $h b b e 2$ and $h b a a 1$ are specifically expressed in larvae and adults, respectively [68]. Quantitative PCR analyses demonstrated decreased hbaal and hbbe1 expression, but increased hbae1, hbae3, hbbe2, and hbbe3 expression to varying degrees (Additional file 14: Figure S7). Given the reduced availability of heme and iron in Rpl11-deficient zebrafish, we deduced that translation of globins might depend on the availability of heme [69]. Because RPL11 deficiency specifically impairs the translation of erythroid genes in murine and human erythroblasts [26], we hypothesized that Rpl11 may specifically inhibit erythroid-specific globin translation in zebrafish embryos.

Dysfunction of iron- and heme-metabolism related genes in Rpl11-deficient zebrafish

Erythrocyte hemoglobinization involves upregulation and close integration of the heme synthesis and iron

Table 3 Summary of affected iron metabolism-related genes in Rpl11-deficient zebrafish embryos

\begin{tabular}{|c|c|c|c|c|c|}
\hline Zebrafish gene & Fold change & Functions & $\begin{array}{l}\text { Hematopoiesis-associated } \\
\text { phenotypes }\end{array}$ & Human homolog & Reference \\
\hline alas2 & $\downarrow 1.44$ & First enzyme of heme synthesis & X-linked sideroblastic anemia & ALAS2 & {$[51]$} \\
\hline $\mathrm{tfa}$ & $\downarrow 1.89$ & $\mathrm{Fe}(\mathrm{III})$-carrier in plasma & Severe anemia & TF & {$[52]$} \\
\hline $\mathrm{tfr} 1 \mathrm{~b}$ & $\downarrow 2.75$ & Membrane receptor for Fe(II)-TF & $\begin{array}{l}\text { Embryonic lethality and } \\
\text { hematopoietic defect }\end{array}$ & TFRC & {$[53]$} \\
\hline aco2 & $\downarrow 1.3$ & Bind to IREs; Fe sensors & Microcytic anemia & IRP2 & {$[54]$} \\
\hline fth1a & $\downarrow 1.35$ & Prevents Fe toxicity during erythropoiesis & Embryonic lethality & $\mathrm{FTH} 1$ & {$[55,56]$} \\
\hline fth $1 b$ & $\downarrow 1.83$ & & & & \\
\hline slc25a37 & $\downarrow 1.77$ & Iron transport into mitochondria & $\begin{array}{l}\text { Embryonic lethal with profound } \\
\text { anemia; hypochromic anemia }\end{array}$ & SLC25A37 & {$[57,58]$} \\
\hline $\mathrm{cp}$ & $\downarrow 2.88$ & Oxidizes exported $\mathrm{Fe} 2+$ & Hypochromic microcytic anemia & $C P$ & [59] \\
\hline$h t t$ & $\downarrow 1.27$ & Transferrin receptor trafficking & Hypochromic anemia & $\mathrm{HTT}$ & {$[60]$} \\
\hline sfxn1 & $\downarrow 2.19$ & Mitochondrial transport and iron metabolism & Siderocytic anemia & SFXN1 & {$[61]$} \\
\hline zgc:194125 & $\uparrow 2.73$ & Iron homeostasis and heme synthesis & Sideroblastic anemia & FTMT & {$[37,62]$} \\
\hline zgc:109934 & $\uparrow 2.36$ & & & & \\
\hline zgc:173594 & $\uparrow 4.12$ & & & & \\
\hline
\end{tabular}

The arrow up and down respectively stand for up- and down-regulation of genes in Rpl11-deficient zebrafish embryos. 


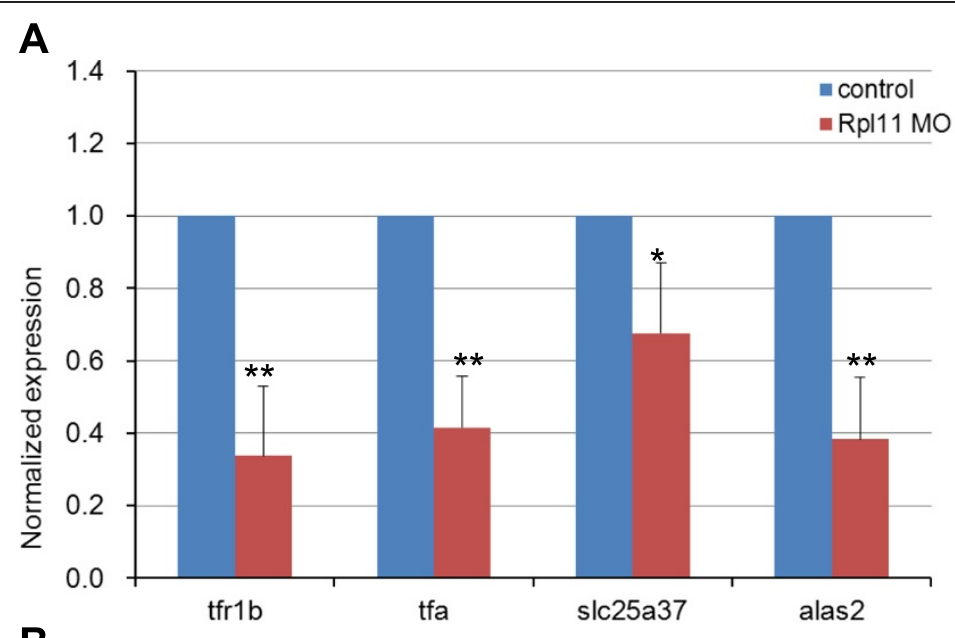

B

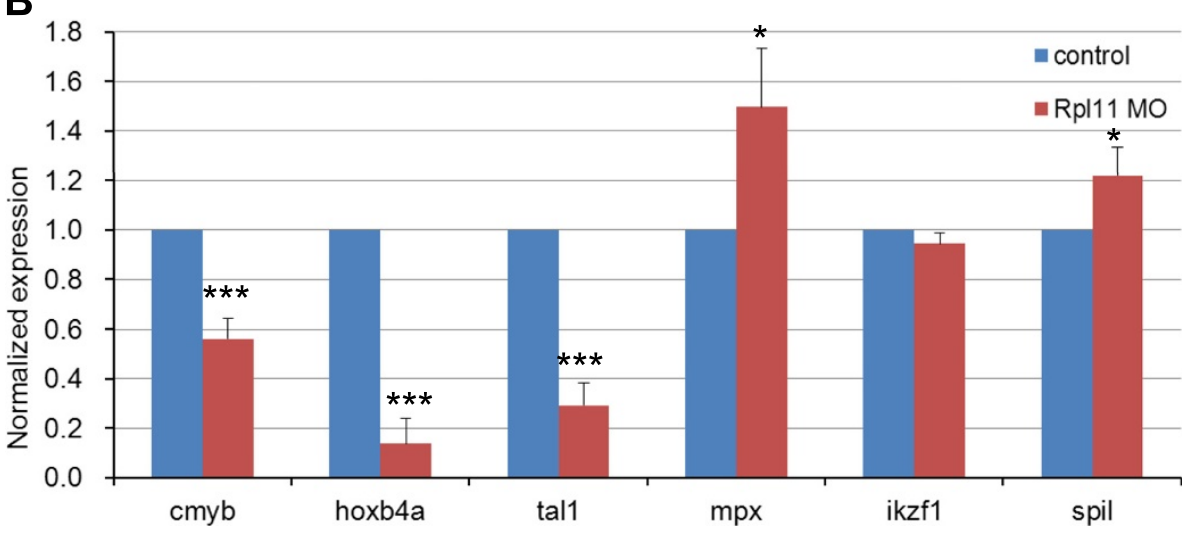

Figure 3 qPCR analysis of changes in the expression of genes potentially associated with hematological defects in Rpl11-deficient zebrafish embryos. A, Analysis of changes in expression of genes associated with iron and heme metabolism in Rpl11-deficient zebrafish embryos; $\mathbf{B}$, Analysis of changes in expression of molecular blood cell lineage markers. Gene expression was represented as mean \pm SD and One-way ANOVA was performed for comparison between $\mathrm{MO}$ control and Rpl11-deficient embryos $\left({ }^{* * *} \mathrm{P}<0.001,{ }^{* *} \mathrm{P}<0.01,{ }^{*}<0.05, \mathrm{n}=3\right.$ ). Gene expression in MO control samples was normalized to 1.

supply pathways during hematopoiesis. In Rpl11deficient zebrafish, the vast majority of iron and heme metabolism-related genes were downregulated, contributing significantly to defective hemoglobinization and hematopoiesis. Hematopoiesis requires large amounts of iron. In serum, most iron is bound to transferrin (Tf; tfa in zebrafish), which directs iron to hemoglobin via transferrin receptor (TfR1; tfr1b in zebrafish)-mediated endocytosis $[70,71]$. TfR1 (tfr1b) is highly expressed in erythrocytes and is directly associated with hemoglobin synthesis and erythroid progenitor maturation $[71,72]$. On binding, the $\mathrm{Fe}(\mathrm{III})$-transferrin-TfR1 complex is rapidly internalized, and iron atoms are released and directed to the mitochondria for heme synthesis [70,71]. The mitochondrial iron transporter SLC25A37 (slc25a37 in zebrafish) is also highly expressed in erythrocytes, and its activity has been shown to be rate limiting for heme synthesis in erythrocytes [57,58]. Moreover, deficiency of the Drosophila homolog mfrn causes a $90 \%$ decrease in heme synthesis, and knockout of Slc25a37 in mice leads to embryonic lethality with profound anemia [58]. Zebrafish with the mutant slc25a37 show severe hypochromic anemia due to defective mitochondrial iron uptake [57]. ALAS2 is an erythroid-specific rate-limiting enzyme responsible for synthesis of protoporphyrin IX, which is transported out of the mitochondria and installed in globin chains for hemoglobin biosynthesis [73]. Indeed, mutations of Alas2 in mice caused sideroblastic anemia [51]. Finally, FTMT was not expressed in normal erythroblasts but was highly expressed in patients with sideroblastic anemia [62]. Upregulation of FTMT results in accumulation of mitochondrial iron followed by disruption of intracellular iron homeostasis [36]. Moreover, overexpression of FTMT limits the availability of iron for heme synthesis and impairs mitochondrial iron metabolism [37]. We also observed increased expression of the putative zebrafish FTMT homologs $z g c: 194125$, zgc:109934, and $z g c: 173594$ in Rpl11-deficient zebrafish embryos, further indicating hematopoiesis-associated disease exists in Rpl11-deficient zebrafish. Taken together, these observations suggest that 


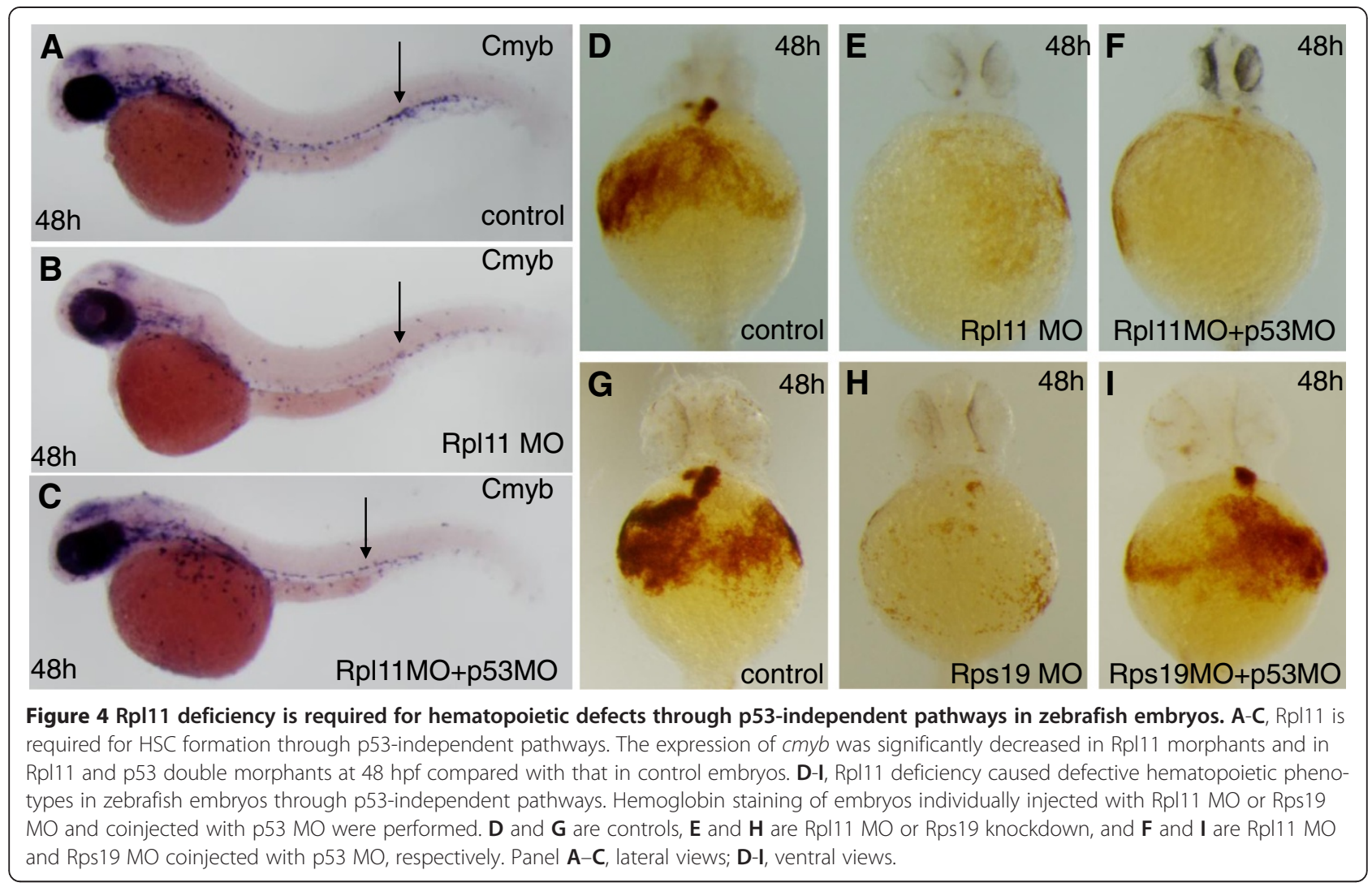

Rpl11 participates in zebrafish hematopoiesis as a regulator of iron and heme metabolism.

\section{Rpl11-deficient zebrafish could serve as a human DBA model}

RPL11 gene mutation leads to disease phenotypes of human DBA, including hematopoietic defects and physical deformities [18]. In zebrafish, Rpl11 deficiency leads to hematopoietic and metabolic defects [8] as well as brain developmental abnormalities [31]. Zebrafish with dysfunctional RPs have been regarded as suitable animal models for DBA studies [30]. Using the Rpl11-deficient zebrafish model and transcriptome deep sequencing, we have added crucial details to the understanding of the biological functions of Rpl11 in zebrafish and have elucidated potential mechanisms by which Rpl11 regulates hematopoiesis. Future studies will determine which pathways and regulatory mechanisms are common to both human DBAs and this zebrafish model. Zebrafish may serve as model systems for human diseases, and hence, our findings provide an important resource and shed new insights for the study of human DBA diseases.

\section{Conclusion}

In this study, we established an Rpl11-deficient zebrafish model that showed defective hemoglobinization and hematopoiesis. To elucidate the mechanisms of hematopoietic failure, we performed mRNA transcriptome deep sequencing of Rpl11-deficient and control MO zebrafish embryos and systematically characterized Rpl11-mediated disturbances in hematological gene expression, hematopoiesisrelated signaling pathways, and regulatory networks in zebrafish. We also elucidated potential mechanisms by which Rpl11 regulates hematopoiesis through genes associated with iron metabolism and proliferation and development of HSCs in zebrafish embryos or through hematopoiesis-associated pathways with unknown mechanisms (Figure 5). The present data provide new insights into the pathogenesis of RPL11-mediated DBA.

\section{Methods}

Zebrafish embryo maintenance, Rpl11 morpholino (MO) microinjection, and hemoglobin staining

Wild-type zebrafish (Danio rerio; $A B$ type) were maintained under standard library conditions and zebrafish embryo stages (hours post fertilization; hpf) were determined as described previously [74,75]. All studies of zebrafish were approved by the Animal Care and Use Committee of Huazhong University of Science and Technology. Rpl11 Morphlino (MO; 5-CTTCTTCTCGCTCTGGTCCGCCA TG-3) and control MO (5-CTTATTCGCGCTATGGTC GGCAATG-3) were obtained from Gene-Tools, LLC. The Rps19 MO, p53 MO, and control MO were previously described [76]. Zebrafish embryos at the one-cell stage were 


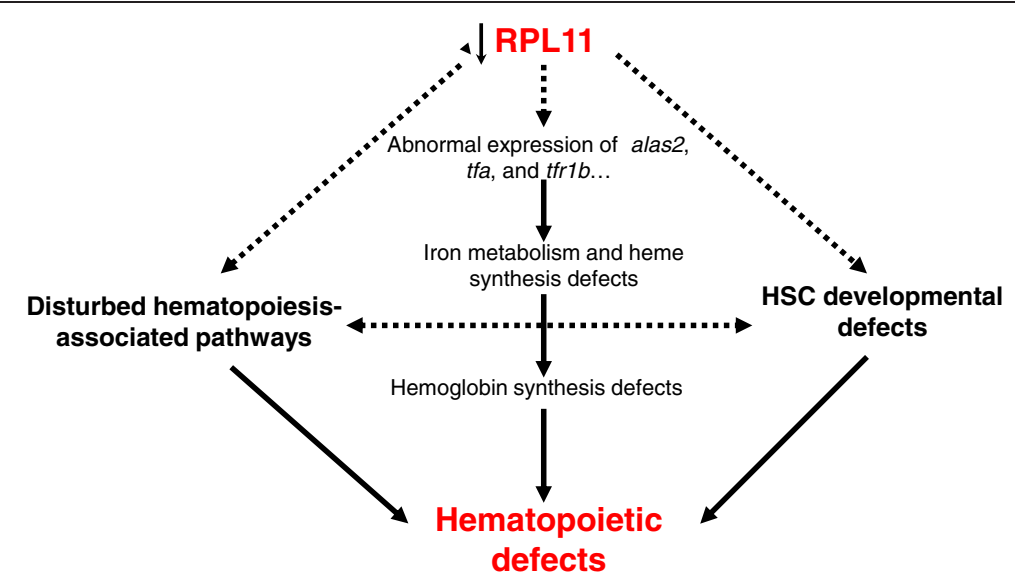

Figure 5 Potential molecular mechanisms by which Rpl11 deficiency leads to hematopoietic defects in zebrafish embryos. In this study, we deduced three potential mechanisms by which Rpl11 deficiency leads to hematopoietic defects in zebrafish embryos. First, Rpl11 deficiency led to abnormal expression of a set of iron metabolism- and heme synthesis-related genes. Second, Rpl11 deficiency disturbed proliferation and development of HSCs. Finally, Rpl11 deficiency disturbed several hematopoiesis-associated pathways. Solid lines represent mechanisms that have been confirmed in this or other studies, and dashed lines represent mechanisms that require further validation.

injected with MOs using a microinjector (WPI SYSPV830). Based on the literature and our initial injection trials, 0.5-ng Rpl11 MO and control MO were chosen as the optimal concentration. The effectiveness of translational inhibition by this Rpl11 MO was tested in vivo using a Rpl11-egfp green fluorescent fusion protein. We found that at a dose of $0.5 \mathrm{ng} /$ embryo, most GFP expression was knocked down. Hemoglobin in zebrafish embryos was analyzed using o-dianisidine (Sigma) as described [77]. All images were collected using an Olympus microscope with a digital camera (OLYMPUS IX71) and were imported into Adobe Photoshop CS2 9.0.2 for orientation and figure preparation.

Total RNA isolation, library preparation, and sequencing Immediately after harvesting, 40-50 pooled embryos at $48 \mathrm{hpf}$ from at least three experiment were snap-frozen in liquid nitrogen and stored at $-80^{\circ} \mathrm{C}$. Total RNA was extracted from pooled embryos using TRIzol reagent (Invitrogen) according to the manufacturer's instructions. RNA concentrations were determined using NanoDrop 2000 (Thermo Scientific). The integrity of RNA samples was determined using $1.2 \%$ agarose gel electrophoresis, followed by removal of the residual genomic DNA with RNase-free DNase I (Ambion). Libraries of mRNA were constructed using the Illumina mRNA-Seq library preparation kit according to the manufacturer's instructions. Concentration and size distribution of the libraries were determined on an Agilent Bioanalyzer DNA 2000 chip (Agilent Technologies), followed by sequencing on an Illumina Hiseq 2000 Genome Analyser platform in pair-end mode by 100-bp lengths. A total of 35-40 million reads were collected for further analysis.

\section{RNA-Seq data analysis}

Reads were processed and aligned to the UCSC zebrafish reference genome (build $\mathrm{Zv9}$ /danRer7, Jul. 2010) using TopHat (version 1.3.3) [78]. TopHat incorporates the Bowtie v0.12.7 algorithm to perform alignments. In brief, TopHat initially removes a portion of reads based on the quality of information accompanying each read and maps qualified reads to the reference genome. The reference index was built using Bowtie with a fasta file for the entire genome of zebrafish, which was downloaded from UCSC (http://genome.ucsc.edu/). Parameters were set by default, but the number of threads to align reads was set at 6 . TopHat aligned read files were then entered into Cufflinks (version 1.2.1) software for further analyses, including transcript assembly, abundance estimation, and differential expression and regulation testing in RNA-Seq samples [79]. To calculate gene expression intensity, read counts were normalized to the number of fragments per kilobase of transcript per million mapped reads (FPKM) according to the gene length and total mapped reads [78]. Confidence intervals for estimates of FPKM were calculated using a Bayesian inference method [80]. The R package DEGseq was used to identify DEGs from RNA-Seq data. Genes with FPKM less than 1 were removed from analyses. DEGs were characterized according to the criterion of fold change (FC) $>2.0$ and p-value $<0.05$.

\section{RT-PCR}

Real-time RT-PCR was performed to verify the expression of genes detected in mRNA-seq experiments involving Rpl11-deficient and MO control zebrafish embryos. Reverse transcription was performed using the cDNA 
Reverse Transcription Kit (Fermentas) with oligo d(T)s. RT-PCR was performed using SYBR Green PCR Master Mix (Fermentas) with a CFX-96 Real-time PCR system according to the manufacturer's instructions (Bio-Rad) (Additional file 15: Table S10). PCR products were analyzed using agarose gel electrophoresis to identify bands of expected sizes. Gene expression was calculated according to the real-time PCR manual of Bio-Rad and normalized to the expression of $\beta$-actin. Data were represented as mean $\pm \mathrm{SD}$ and One-way ANOVA was performed for comparison between $\mathrm{MO}$ control and Rpl11-deficient embryos.

\section{IPA analysis}

Gene interaction networks and signaling pathways were generated using Ingenuity Pathways Analysis (IPA) software (http://www.ingenuity.com/) from Ingenuity ${ }^{\circ}$ Systems. This software was used to analyze data from a variety of experimental platforms and to provide accurate biological insights into interactions between genes, proteins, chemicals, pathways, cellular phenotypes, and disease processes. Differentially expressed genes were processed using DEGseq and screened according to the criteria FC $>2$ and $\mathrm{p}$-value $<0.05$. These genes were submitted to IPA for biological function, canonical pathway, and interaction network analyses. Since the current edition of IPA cannot accept and identify zebrafish gene IDs, we matched these differentially expressed zebrafish genes with their human homologs using the homolog database HomoloGene, which was downloaded from NCBI. After conversion to human homolog genes, these homologs were submitted to IPA, sets of genes that were enriched for a particular function or pathway were identified, and enrichment ratios were calculated. To determine the significance of enrichment in a particular function, IPA calculates the significance value based on the measure of involvement of the gene in the input data set with its respective molecular functions and signaling pathways [81]. The significance of networks was calculated using Fisher's exact test, and p-values were executed using negative logarithmic transformation.

\section{Screening of differentially expressed hematological genes}

Zebrafish gene sets associated with hematological systems were identified by searching with the keywords "erythroid," "hematopoietic," "globin," and "hema" using the AmiGO software of the Gene Ontology (GO) database (http://www.geneontology.org/). Differentially expressed genes $(\mathrm{FC}>2.0$, $\mathrm{p}$-value $<0.05)$ detected by DEGseq were mapped to this hematological gene set, which indicated the differentially expressed hematological genes in our data set. Mapping and drawing of the scatter diagrams were accomplished using $\mathrm{R}$ scripts.

\section{Cluster analysis}

Expression intensities of differentially expressed hematological genes were used in cluster analyses. Gene expression intensity was normalized using the equation $x_{i}^{\prime}=\frac{x_{i}-\bar{x}}{\bar{x}}$, where $x_{i}$ is the FPKM value and $\bar{x}$ is the mean FPKM value of a gene calculated from two samples. Average linkage hierarchical clustering was performed in this study using Pearson distance as the measure between genes and samples. Computation and visualization were achieved using the heatmap plus package in $\mathrm{R}$.

\section{Data access}

Supporting data sets are available in the Gene Expression Omnibus, with the accession number GSE45699. The following link has been created to allow review of record GSE51326 while it remains in private status: http://www. ncbi.nlm.nih.gov/geo/query/acc.cgi?token=mzcbuyyipbixh un\&acc $=$ GSE51326.

\section{Additional files}

Additional file 1: Table S1. RNA-seq reads mapping analysis.

Additional file 2: Table S2. All gene transcripts detected in Rpl11deficient and $\mathrm{MO}$ control zebrafish embryos using RNA-seq.

Additional file 3: Table S3. All DEGs in Rpl11-deficient zebrafish embryos at $48 \mathrm{hpf}$. This table includes up- and downregulated genes in Rpl11-deficient zebrafish embryos (fold change $>1.5$ or 2 , p-value $<0.05$ ).

Additional file 4: Table S4. Specifically expressed genes in $\mathrm{MO}$ control and Rpl11-deficient zebrafish embryos.

Additional file 5: GO enrichment of up- and downregulated genes in Rpl11-deficient zebrafish embryos at 48 hpf. GO enrichment includes three analytical aspects: biological process, molecular function, and cellular component. DEGs in Rpl11-deficient zebrafish embryos at $48 \mathrm{hpf}$ (FC >2, p-value <0.05) were included in this analysis. Blue labeled functions are associated with DEGs in Rpl11-deficient zebrafish embryos. This figure was generated using AmiGO software (http://www.geneontology. org/). Figure S1, GO enrichment of upregulated genes in Rpl11-deficient zebrafish embryos at $48 \mathrm{hpf}$. Figure S2, GO enrichment of downregulated genes in Rpl11-deficient zebrafish embryos at $48 \mathrm{hpf}$.

Additional file 6: Table S5. Genes involved in GO enrichment of up- and downregulated DEGs in Rpl11-deficient zebrafish embryos.

Additional file 7: Figure S3. APCR analyses of human FTMT homologs in Rpl11-deficient zebrafish embryos at 48 hpf. These genes were zgc:194125, zgc:173594, and zgc:109934, which were specifically enriched in iron metabolism-associated functions such as iron transport and cellular iron homeostasis in GO analyses, and were upregualted in Rpl11-deficient zebrafish embryos (Mean $\pm \mathrm{SD}$, one-way ANOVA, ${ }^{* *} \mathrm{P}<0.01,{ }^{*}<0.05, \mathrm{n}=3$ ). Gene expression in $\mathrm{MO}$ control samples was normalized to 1.

Additional file 8: Figure S4. Scatter plot of DEGs in Rpl11-deficient zebrafish embryos at $48 \mathrm{hpf}$. DEGs affected by Rpl11 deficiency in zebrafish embryos at 48 hpf are shown (FC $>1.5$, p-value $<0.05$ ), and affected hematological genes (highlighted red) were further analyzed.

Additional file 9: Table S6. Affected hematological genes enriched in GO items.

Additional file 10: Table S7. Disturbed signaling pathways in Rpl11deficient zebrafish embryos. This table includes up- and downregulated signaling pathways in Rpl11-deficient zebrafish embryos at $48 \mathrm{hpf}$.

Additional file 11. Networks affected by Rpl11 deficiency in zebrafish embryos at 48 hpf. Networks were generated using online IPA software, 
and highly enriched networks were selected (Score > 20). Genes labeled red and green were up- and downregulated, respectively. Figure S5 (A-C), Upregulated networks affected by Rpl11 deficiency in zebrafish embryos at $48 \mathrm{hpf}$. A, Networks of cellular function and maintenance, small molecule biochemistry, and carbohydrate metabolism. B, Networks of cellular development, cellular growth and proliferation, and connective tissue development and function. C, Networks of cell death and survival, connective tissue disorders, and immunological disease. Figure S6 (A-E), Downregulated networks affected by Rpl11 deficiency in zebrafish embryos at 48 hpf. A, Networks of developmental disorders, skeletal and muscular disorders, and digestive system development and function. B, Networks of cellular development, visual system development and function, nervous system development and function, cellular growth and proliferation, connective tissue development and function, neurological disease, and tissue morphology. C, Networks of cell death and survival, cardiac pulmonary embolism, and cardiovascular disease. D, Networks of cancer, cell cycle, and tissue morphology. E, Networks of cell cycle, cell morphology, and cell-to-cell signaling and interaction.

Additional file 12: Table S8. Genes involved in regulatory interacting networks disturbed in Rpl11-deficiet zebrafish embryos.

Additional file 13: Table S9. Description of blood cell lineage markers. Description of blood cell lineage markers, including cmyb, hoxb4a, tall, $m p x$, spil, and ikzf1, were shown. Blood cell lineages, including hematopoietic stem cells, granulocytes, myelocytes, and lymphoid progenitors were detected in this study. Fold change represents FPKM in Rpl11-deficient zebrafish embryos compared to that of MO control.

Additional file 14: Figure S7. RT-PCR analyses of changes in the expression of globin genes in zebrafish embryos after Rpl11 knockdown. A, RT-PCR analysis of changes in expression of globin genes in zebrafish embryos after Rpl11 knockdown (Mean $\pm \mathrm{SD}$, one-way ANOVA, **P $<0.01$, $\left.{ }^{*}<0.05, n=3\right)$. Gene expression in $\mathrm{MO}$ control samples was normalized to $1 ; B$, Description of globin genes detected in deep sequencing samples.

Additional file 15: Table S10. RT-PCR primers used in this study.

\section{Abbreviations}

DBA: Diamond-Blackfan anemia; Rpl11: Ribosomal protein L11; HSC: Hematopoietic stem cells; FPKM: The number of fragments per kilobase of transcript per million mapped reads; DEGs: Differentially expressed genes; RPs: Ribosomal proteins.

\section{Competing interests}

The authors declare that they have no competing interests.

\section{Authors' contributions}

ZZ was involved in mRNA-Seq library preparation, carried out bioinformatics studies and data organization, and drafted the manuscript. HJ established Rpl11-deficient zebrafish model, carried out zebrafish related experiments, and participated in data organization. QZ participated in the sequence alignment and bioinformatics analysis. YW, YZ, QJ and WZ participated in zebrafish related experiments. WY and TC participated in its overall design and instruction, and revised it critically for important intellectual content. XF and $X Z$ conceived of the study, participated in coordination, and gave final approval of the version to be published. All authors read and approved the final manuscript.

\footnotetext{
Acknowledgements

This work was supported by the "Strategic Priority Research Program" of the Chinese Academy of Sciences, Stem Cell and Regenerative Medicine Research (XDA01040405); National "Twelfth Five-Year" Plan for Science \& Technology Support (2013BAI01B09); the following funds from the Ministry of Science and Technology (2013CB945300, 2012CB966603, 2013CB966902, 2010CB945204, 2012AA022502); NSFC (81090414, 81170470, 81130074, 31171387, 31000640, 81070390, 31371300, 31100924); the National Key Scientific Instrument and Equipment Development Projects of China (2011YQ03013404); Hubei National Natural Science Foundation Grant $2010 C D B 02402$ and State Key Laboratory of Experimental Hematology Pilot Project Grant (ZK12-05, ZK13-05).
}

\section{Author details}

'CAS Key Laboratory of Genome Sciences and Information, Beijing Institute of Genomics, Chinese Academy of Sciences, Beijing 100101, China. ${ }^{2}$ Key Laboratory of Molecular Biophysics of Ministry of Education, College of Life Science and Technology Center for Human Genome Research, Huazhong University of Science and Technology, Wuhan, Hubei 430074, China. ${ }^{3}$ State Key Laboratory of Experimental Hematology, Institute of Hematology and Blood Disease Hospital, Chinese Academy of Medical Sciences \& Peking Union Medical College, Tianjin 300020, China. ${ }^{4}$ Hepatic Surgery Center Tongji Hospital, Tongji Medical College, Huazhong University of Science and Technology, Wuhan, Hubei 430074, China.

Received: 20 May 2013 Accepted: 10 December 2013 Published: 17 December 2013

\section{References}

1. Narla A, Ebert BL: Ribosomopathies: human disorders of ribosome dysfunction. Blood 2010, 115(16):3196-3205.

2. Lipton JM, Ellis SR: Diamond Blackfan anemia 2008-2009: broadening the scope of ribosome biogenesis disorders. Curr Opin Pediatr 2010, 22(1):12-19.

3. Ganapathi KA, Shimamura A: Ribosomal dysfunction and inherited marrow failure. Br J Haematol 2008, 141(3):376-387.

4. Sieff CA, Yang J, Merida-Long LB, Lodish HF: Pathogenesis of the erythroid failure in Diamond Blackfan anaemia. Br J Haematol 2010, 148(4):611-622.

5. Deisenroth C, Zhang Y: The ribosomal protein-Mdm2-p53 pathway and energy metabolism bridging the Gap between feast and famine. Genes Cancer 2011, 2(4):392-403.

6. Dutt S, Narla A, Lin K, Mullally A, Abayasekara N, Megerdichian C, Wilson FH, Currie T, Khanna-Gupta A, Berliner N, et al: Haploinsufficiency for ribosomal protein genes causes selective activation of p53 in human erythroid progenitor cells. Blood 2011, 117(9):2567-2576.

7. Hamaguchi I, Ooka A, Brun A, Richter J, Dahl N, Karlsson S: Gene transfer improves erythroid development in ribosomal protein S19-deficient Diamond-Blackfan anemia. Blood 2002, 100(8):2724-2731.

8. Danilova N, Sakamoto KM, Lin S: Ribosomal protein L11 mutation in zebrafish leads to haematopoietic and metabolic defects. Br J Haematol 2011, 152(2):217-228.

9. Duan J, Ba Q, Wang Z, Hao M, Li X, Hu P, Zhang D, Zhang R, Wang H: Knockdown of ribosomal protein S7 causes developmental abnormalities via p53 dependent and independent pathways in zebrafish. Int J Biochem Cell Biol 2011, 43(8):1218-1227.

10. Taylor AM, Humphries JM, White RM, Murphey RD, Burns CE, Zon LI: Hematopoietic defects in rps29 mutant zebrafish depend upon p53 activation. Exp Hematol 2012, 40(3):228-237.

11. Zhang Y, Duc A-CE, Rao S, Sun X-L, Bilbee AN, Rhodes M, Li Q, Kappes DJ, Rhodes J, Wiest DL: Control of hematopoietic stem cell emergence by antagonistic functions of ribosomal protein paralogs. Dev Cell 2013, 24(4):411-425.

12. Ball SE, McGuckin CP, Jenkins G, Gordon-Smith EC: Diamond-Blackfan anaemia in the U.K.: analysis of 80 cases from a 20 -year birth cohort. BrJ Haematol 1996, 94(4):645-653.

13. Lipton JM, Atsidaftos E, Zyskind I, Vlachos A: Improving clinical care and elucidating the pathophysiology of Diamond Blackfan anemia: an update from the Diamond Blackfan Anemia Registry. Pediatr Blood Cancer 2006, 46(5):558-564.

14. Ellis SR, Gleizes PE: Diamond Blackfan anemia: ribosomal proteins going rogue. Semin Hematol 2011, 48(2):89-96.

15. Boria I, Garelli E, Gazda HT, Aspesi A, Quarello P, Pavesi E, Ferrante D, Meerpohl JJ, Kartal M, Da Costa L, et al: The ribosomal basis of Diamond-Blackfan Anemia: mutation and database update. Hum Mutat 2010, 31(12):1269-1279.

16. Da Costa L, Moniz H, Simansour M, Tchernia G, Mohandas N, Leblanc T: Diamond-Blackfan anemia, ribosome and erythropoiesis. Transfus Clin Biol 2010, 17(3):112-119.

17. Gazda HT, Sheen MR, Vlachos A, Choesmel V, O'Donohue MF, Schneider H, Darras N, Hasman C, Sieff CA, Newburger PE, et al: Ribosomal protein L5 and L11 mutations are associated with cleft palate and abnormal thumbs in Diamond-Blackfan anemia patients. Am J Hum Genet 2008, 83(6):769-780.

18. Cmejla R, Cmejlova J, Handrkova H, Petrak J, Petrtylova K, Mihal V, Stary J, Cerna Z, Jabali Y, Pospisilova D: Identification of mutations in the 
ribosomal protein L5 (RPL5) and ribosomal protein L11 (RPL11) genes in Czech patients with Diamond-Blackfan anemia. Hum Mutat 2009, 30(3):321-327

19. Quarello P, Garelli E, Carando A, Brusco A, Calabrese R, Dufour C, Longoni D, Misuraca A, Vinti L, Aspesi A: Diamond-Blackfan anemia: genotype-phenotype correlations in Italian patients with RPL5 and RPL11 mutations. Haematologica 2010, 95(2):206-213.

20. Gazda HT, Grabowska A, Merida-Long LB, Latawiec E, Schneider HE, Lipton JM, Vlachos A, Atsidaftos E, Ball SE, Orfali KA: Ribosomal protein S24 gene is mutated in Diamond-Blackfan anemia. Am J Hum Genet 2006, 79(6):1110-1118.

21. Cmejla R, Cmejlova J, Handrkova H, Petrak J, Pospisilova D: Ribosomal protein $\mathrm{S} 17$ gene (RPS17) is mutated in Diamond-Blackfan anemia. Hum Mutat 2007, 28(12):1178-1182.

22. Farrar JE, Nater M, Caywood E, McDevitt MA, Kowalski J, Takemoto CM Talbot CC Jr, Meltzer P, Esposito D, Beggs AH: Abnormalities of the large ribosomal subunit protein, Rpl35A, in diamond-blackfan anemia. Blood 2008, 112(5):1582-1592.

23. Doherty L, Sheen MR, Vlachos A, Choesmel V, O'donohue MF, Clinton C, Schneider HE, Sieff CA, Newburger PE, Ball SE: Ribosomal protein genes RPS10 and RPS26 are commonly mutated in Diamond-Blackfan anemia. Am J Hum Genet 2010, 86(2):222-228.

24. Draptchinskaia N, Gustavsson P, Andersson B, Pettersson M: The gene encoding ribosomal protein $\mathrm{S} 19$ is mutated in Diamond-Blackfan anaemia. Nat Genet 1999, 21(2):169-175.

25. Moniz H, Gastou M, Leblanc T, Hurtaud C, Crétien A, Lécluse Y, Raslova H, Larghero J, Croisille L, Faubladier M: Primary hematopoietic cells from DBA patients with mutations in RPL11 and RPS19 genes exhibit distinct erythroid phenotype in vitro. Cell Death Dis 2012, 3(7):e356.

26. Horos R, ljspeert H, Pospisilova D, Sendtner R, Andrieu-Soler C, Taskesen E, Nieradka A, Cmejla R, Sendtner M, Touw IP, et al: Ribosomal deficiencies in Diamond-Blackfan anemia impair translation of transcripts essential for differentiation of murine and human erythroblasts. Blood 2012, 119 (1):262-272.

27. Storer NY, Zon LI: Zebrafish Models of p53 Functions. Cold Spring Harb Perspect Biol 2010, 2(8):a001123.

28. Jing L, Zon LI: Zebrafish as a model for normal and malignant hematopoiesis. Dis Model Mech 2011, 4(4):433-438.

29. Uechi T, Nakajima Y, Nakao A, Torihara H, Chakraborty A, Inoue K, Kenmochi $\mathrm{N}$ : Ribosomal protein gene knockdown causes developmental defects in zebrafish. PLoS One 2006, 1(1):e37.

30. Torihara H, Uechi T, Chakraborty A, Shinya M, Sakai N, Kenmochi N: Erythropoiesis failure due to RPS19 deficiency is independent of an activated Tp53 response in a zebrafish model of Diamond-Blackfan anaemia. Br J Haematol 2011, 152(5):648-654.

31. Chakraborty A, Uechi T, Higa S, Torihara H, Kenmochi N: Loss of ribosomal protein L11 affects zebrafish embryonic development through a p53-dependent apoptotic response. PLoS One 2009, 4(1):e4152

32. Provost E, Wehner KA, Zhong X, Ashar F, Nguyen E, Green R, Parsons MJ, Leach SD: Ribosomal biogenesis genes play an essential and p53-independent role in zebrafish pancreas development. Development 2012, 139(17):3232-3241.

33. Vesterlund L, Jiao H, Unneberg $P$, Hovatta O, Kere J: The zebrafish transcriptome during early development. BMC Dev Bio/ 2011, 11(1):30.

34. Aanes $\mathrm{H}$, Winata $\mathrm{CL}$, Lin $\mathrm{CH}$, Chen JP, Srinivasan $\mathrm{KG}$, Lee $\mathrm{SG}$, Lim AY, Hajan HS, Collas P, Bourque G: Zebrafish mRNA sequencing deciphers novelties in transcriptome dynamics during maternal to zygotic transition. Genome Res 2011, 21(8):1328-1338.

35. Mortazavi A, Williams BA, McCue K, Schaeffer L, Wold B: Mapping and quantifying mammalian transcriptomes by RNA-Seq. Nat Methods 2008, 5(7):621-628.

36. Nie G, Sheftel AD, Kim SF, Ponka P: Overexpression of mitochondrial ferritin causes cytosolic iron depletion and changes cellular iron homeostasis. Blood 2005, 105(5):2161-2167.

37. Richardson DR, Lane DJR, Becker EM, Huang MLH, Whitnall M, Rahmanto YS, Sheftel AD, Ponka P: Mitochondrial iron trafficking and the integration of iron metabolism between the mitochondrion and cytosol. Proc Natl Acad Sci U S A 2010, 107(24):10775-10782.

38. Bauer A, Tronche F, Wessely O, Kellendonk C, Reichardt HM, Steinlein P, Schutz G, Beug H: The glucocorticoid receptor is required for stress erythropoiesis. Genes Dev 1999, 13(22):2996-3002.
39. Flygare J, Rayon Estrada V, Shin C, Gupta S, Lodish HF: HIF1 alpha synergizes with glucocorticoids to promote BFU-E progenitor self-renewal. Blood 2011, 117(12):3435-3444

40. Correa PN, Axelrad AA: Production of erythropoietic bursts by progenitor cells from adult human peripheral blood in an improved serum-free medium: role of insulinlike growth factor 1. Blood 1991, 78(11):2823-2833.

41. Klusmann JH, Godinho FJ, Heitmann K, Maroz A, Koch ML, Reinhardt D, Orkin SH, Li Z: Developmental stage-specific interplay of GATA1 and IGF signaling in fetal megakaryopoiesis and leukemogenesis. Genes Dev 2010, 24(15):1659-1672.

42. Ratajczak J, Zhang Q, Pertusini E, Wojczyk B, Wasik M, Ratajczak M: The role of insulin (INS) and insulin-like growth factor-I (IGF-I) in regulating human erythropoiesis. Studies in vitro under serum-free conditions: comparison to other cytokines and growth factors. Leukemia 1998, 12(3):371-381.

43. Krstic A, Mojsilovic S, Jovcic G, Bugarski D: The potential of interleukin-17 to mediate hematopoietic response. Immunol Res 2012, 52(1-2):34-41.

44. Marks-Bluth J, Pimanda JE: Cell signalling pathways that mediate haematopoietic stem cell specification. Int J Biochem Cell Biol 2012, 44(12):2175-2184.

45. Luis TC, Naber BAE, Roozen PPC, Brugman MH, de Haas EFE, Ghazvini M, Fibbe WE, van Dongen JJM, Fodde R, Staal FJT: Canonical Wnt signaling regulates hematopoiesis in a dosage-dependent fashion. Cell Stem Cell 2011, 9(4):345-356

46. Ruiz-Herguido C, Guiu J, D'Altri T, Ingles-Esteve J, Dzierzak E, Espinosa L, Bigas A: Hematopoietic stem cell development requires transient Wnt/ beta-catenin activity. J Exp Med 2012, 209(8):1457-1468.

47. Tran HT, Sekkali B, Van Imschoot G, Janssens S, Vleminckx K: Wnt/ $\beta$-catenin signaling is involved in the induction and maintenance of primitive hematopoiesis in the vertebrate embryo. Proc Natl Acad Sci USA 2010, 107(37):16160-16165.

48. Zhang C, Patient R, Liu F: Hematopoietic stem cell development and regulatory signaling in zebrafish. Biochim Biophys Acta 2012, 1830(2):2370-2374.

49. Singh KP, Garrett RW, Casado FL, Gasiewicz TA: Aryl hydrocarbon receptornull allele mice have hematopoietic stem/progenitor cells with abnormal characteristics and functions. Stem Cells Dev 2010, 20(5):769-784.

50. Wingert RA, Galloway JL, Barut B, Foott H, Fraenkel P, Axe JL, Weber GJ, Dooley K, Davidson AJ, Schmid B, et al: Deficiency of glutaredoxin 5 reveals Fe-S clusters are required for vertebrate haem synthesis. Nature 2005, 436(7053):1035-1039.

51. Bottomley SS, May BK, Cox TC, Cotter PD, Bishop DF: Molecular defects of erythroid 5-aminolevulinate synthase in X-linked sideroblastic anemia. J Bioenerg Biomembr 1995, 27(2):161-168.

52. Roy CN, Weinstein DA, Andrews NC: 2002 E. Mead Johnson award for research in pediatrics lecture: the molecular biology of the anemia of chronic disease: a hypothesis. Pediatr Res 2003, 53(3):507-512.

53. Levy JE, Jin O, Fujiwara Y, Kuo F, Andrews NC: Transferrin receptor is necessary for development of erythrocytes and the nervous system. Nat Genet 1999, 21(4):396-399.

54. Cooperman SS, Meyron-Holtz EG, Olivierre-Wilson H, Ghosh MC, McConnell JP, Rouault TA: Microcytic anemia, erythropoietic protoporphyria, and neurodegeneration in mice with targeted deletion of iron-regulatory protein 2. Blood 2005, 106(3):1084-1091.

55. Ferreira C, Bucchini D, Martin ME, Levi S, Arosio P, Grandchamp B, Beaumont $C$ : Early embryonic lethality of $\mathrm{H}$ ferritin gene deletion in mice. J Biol Chem 2000, 275(5):3021-3024

56. Thompson K, Menzies S, Muckenthaler M, Torti FM, Wood T, Torti SV, Hentze MW, Beard J, Connor J: Mouse brains deficient in $\mathrm{H}$-ferritin have normal iron concentration but a protein profile of iron deficiency and increased evidence of oxidative stress. J Neurosci Res 2003, 71(1):46-63.

57. Shaw GC, Cope JJ, Li L, Corson K, Hersey C, Ackermann GE, Gwynn B, Lambert AJ, Wingert RA, Traver D: Mitoferrin is essential for erythroid iron assimilation. Nature 2006, 440(7080):96-100.

58. Paradkar PN, Zumbrennen KB, Paw BH, Ward DM, Kaplan J: Regulation of mitochondrial iron import through differential turnover of mitoferrin 1 and mitoferrin 2. Mol Cell Biol 2009, 29(4):1007-1016.

59. Jeong SY, David S: Age-related changes in iron homeostasis and cell death in the cerebellum of ceruloplasmin-deficient mice. J Neurosci 2006, 26(38):9810-9819.

60. Lumsden AL, Henshall TL, Dayan S, Lardelli MT, Richards Rl: Huntingtindeficient zebrafish exhibit defects in iron utilization and development. Hum Mol Genet 2007, 16(16):1905-1920. 
61. Fleming MD, Campagna DR, Haslett JN, Trenor CC, Andrews NC: A mutation in a mitochondrial transmembrane protein is responsible for the pleiotropic hematological and skeletal phenotype of flexed-tail (f/f) mice. Genes Dev 2001, 15(6):652-657.

62. Cazzola M, Invernizzi R, Bergamaschi G, Levi S, Corsi B, Travaglino E, Rolandi V, Biasiotto G, Drysdale J, Arosio P: Mitochondrial ferritin expression in erythroid cells from patients with sideroblastic anemia. Blood 2003, 101(5):1996-2000.

63. Antonchuk J, Sauvageau G, Humphries RK: HOXB4-induced expansion of adult hematopoietic stem cells ex vivo. Cell 2002, 109(1):39-45.

64. Wilson NK, Miranda-Saavedra D, Kinston S, Bonadies N, Foster SD, Calero-Nieto F, Dawson MA, Donaldson IJ, Dumon S, Frampton J: The transcriptional program controlled by the stem cell leukemia gene $\mathrm{Scl} / \mathrm{Tal} 1$ during early embryonic hematopoietic development. Blood 2009, 113(22):5456-5465.

65. Fumagalli S, Thomas G: The role of p53 in ribosomopathies. Seminars in hematology 2011, 48(2):97-105.

66. Maclnnes AW, Amsterdam A, Whittaker CA, Hopkins N, Lees JA: Loss of p53 synthesis in zebrafish tumors with ribosomal protein gene mutations. Proc Natl Acad Sci U S A 2008, 105(30):10408-10413.

67. Lipton JM, Federman N, Khabbaze Y, Schwartz CL, Hilliard LM, Clark JI, Vlachos A: Osteogenic sarcoma associated with Diamond-Blackfan anemia: a report from the Diamond-Blackfan Anemia Registry. J Pediatr Hematol Oncol 2001, 23(1):39-44.

68. Ganis JJ, Hsia N, Trompouki E, de Jong JLO, DiBiase A, Lambert JS, Jia ZY Sabo PJ, Weaver M, Sandstrom R, et al: Zebrafish globin switching occurs in two developmental stages and is controlled by the LCR. Dev Biol 2012, 366(2):185-194.

69. Chen JJ: Regulation of protein synthesis by the heme-regulated elF2alpha kinase: relevance to anemias. Blood 2007, 109(7):2693-2699.

70. Ponka P: Tissue-specific regulation of iron metabolism and heme synthesis: distinct control mechanisms in erythroid cells. Blood 1997, 89(1):1-25.

71. De Domenico I, Ward DM, Kaplan J: Regulation of iron acquisition and storage: consequences for iron-linked disorders. Nat Rev Mol Cell Biol 2008, 9(1):72-81.

72. Hentze MW, Muckenthaler MU, Andrews NC: Balancing acts: molecular control of mammalian iron metabolism. Cell 2004, 117(3):285-297.

73. Furuyama K, Kaneko K, Vargas VPD: Heme as a magnificent molecule with multiple missions: heme determines its own fate and governs cellular homeostasis. Tohoku J Exp Med 2007, 213(1):1-16.

74. Westerfield M, Doerry E, Douglas S: Zebrafish in the Net. Trends Genet 1999, 15(6):248-249

75. Kimmel CB, Ballard WW, Kimmel SR, Ullmann B, Schilling TF: Stages of embryonic development of the zebrafish. Dev Dyn 1995, 203(3):253-310.

76. Jia Q, Zhang Q, Zhang ZJ, Wang YQ, Zhang WG, Zhou Y, Wan Y, Cheng T, Zhu XF, Fang XD, et al: Transcriptome analysis of the zebrafish model of diamond-blackfan anemia from RPS19 deficiency via p53-dependent and -independent pathways. Plos One 2013, 8(8):e71782.

77. Hr D, Kieran MW, Chan FY, Barone LM, Yee K, Rundstadler JA, Pratt S, Ransom D, Zon LI: Intraembryonic hematopoietic cell migration during vertebrate development. Proc Natl Acad Sci U S A 1995, 92(23):10713-10717.

78. Trapnell C, Williams BA, Pertea G, Mortazavi A, Kwan G, van Baren MJ, Salzberg SL, Wold BJ, Pachter L: Transcript assembly and quantification by RNA-Seq reveals unannotated transcripts and isoform switching during cell differentiation. Nat Biotechnol 2010, 28(5):511-515.

79. Roberts A, Trapnell C, Donaghey J, Rinn JL, Pachter L: Improving RNA-Seq expression estimates by correcting for fragment bias. Genome Biol 2011, 12(3):R22.

80. Jiang $\mathrm{H}$, Wong WH: Statistical inferences for isoform expression in RNA-Seq. Bioinformatics 2009, 25(8):1026-1032.

81. Hegde A, Qiu NC, Qiu X, Ho SH, Tay KQ, George J, Ng FS, Govindarajan KR, Gong Z, Mathavan S, et al: Genomewide expression analysis in zebrafish mind bomb alleles with pancreas defects of different severity identifies putative Notch responsive genes. PLoS One 2008, 3(1):e1479.

doi:10.1186/1471-2164-14-896

Cite this article as: Zhang et al:: Assessment of hematopoietic failure due to Rpl11 deficiency in a zebrafish model of Diamond-Blackfan anemia by deep sequencing. BMC Genomics 2013 14:896.

\section{Submit your next manuscript to BioMed Central and take full advantage of:}

- Convenient online submission

- Thorough peer review

- No space constraints or color figure charges

- Immediate publication on acceptance

- Inclusion in PubMed, CAS, Scopus and Google Scholar

- Research which is freely available for redistribution 